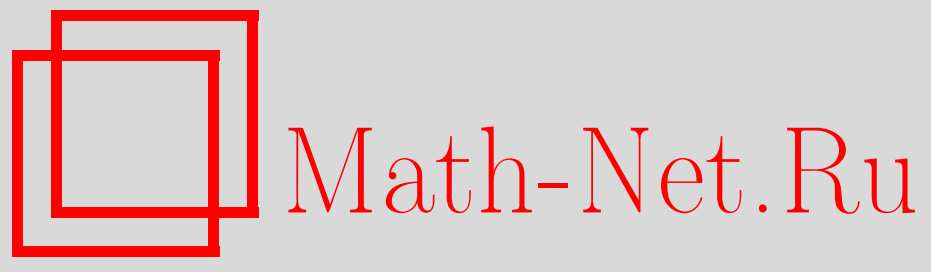

Р. Я. Будылин, Неравенство, полученное при рассмотрении нестабильных решеток ранга 2, УМH, 2011, том 66, выпуск 4, 183-184

DOI: https://doi.org/10.4213/rm9430

Использование Общероссийского математического портала Math-Net.Ru подразумевает, что вы прочитали и согласны с пользовательским соглашением http://www .mathnet.ru/rus/agreement

Параметры загрузки:

IP : 3.89 .197 .203

26 апреля 2023 г., 13:18:53

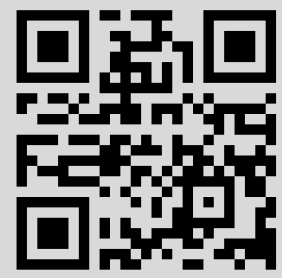




\section{Неравенство, полученное при рассмотрении нестабильных решеток ранга 2}

\section{Р. Я. Будылин}

Пусть $K$ - числовое поле с кольцом целых $F, n=[K: \mathbb{Q}]<\infty$ и $P$ - проективный модуль над кольцом $F$. Поля $K_{i}$ - архимедовы пополнения поля $K$. Пусть $r$ - число вещественных точек, $s$ - комплексных. Мы будем пользоваться определениями из [1].

ОПределение 1 . Структура $F$-решетки на модуле $P$ есть набор скалярных (эрмитовых) произведений на каждом вещественном (комплексном) векторном пространстве $P \otimes_{F} K_{i}$. Если $K_{i}=\mathbb{C}$, то скалярное произведение предполагается эрмитовым.

ОпредЕЛЕние 2. Решетку называют полустабильной, если ее каноническая фильтрация имеет вид $0 \subset P$. В противном случае решетка называется нестабильной.

Пусть $P=F^{k}$. Обозначим через $O\left(k, K_{i}\right)$ группу $O_{k}(\mathbb{R})$ для вещественных точек и группу $U_{k}(\mathbb{C})$ - для комплексных точек. Множество скалярных (эрмитовых) произведений над вещественным (комплексным) пополнением $K_{i}$ отождествляется c $\operatorname{Gl}\left(k, K_{i}\right) / O\left(k, K_{i}\right)$. Обозначим группу $\prod_{K_{i}} \operatorname{Gl}\left(k, K_{i}\right)$ через $G$, группу $\left\{\left(x_{i}\right) \in G \mid\right.$ $\left.\prod_{K_{i}}\left\|x_{i}\right\|=1\right\}$ через $G^{\prime}$, группу $\operatorname{Gl}(k, F)$ через $H$, группу $\prod_{K_{i}} O\left(k, K_{i}\right)$ через $O$. Множество решеток с точностью до эквивалентности отождествляется со множеством $H \backslash G / O$. Множество $G / O$ отождествляется со множеством решеток с выделенным базисом в $F^{n}$. Назовем его элементы вложенными решетками. Элементы множества $G^{\prime} / O$ назовем специальными вложенными решетками. По теореме о сильной аппроксимации действие группы $H$ на множестве $G^{\prime} / O$ - это действие с компактным фактором. Пусть множество $M$ - фундаментальная область этого действия.

Пусть $N\left(k, K_{i}\right)$ - унипотентные матрицы из $\mathrm{Gl}\left(k, K_{i}\right)$, группа $A\left(k, K_{i}\right)$ - вещественные диагональные матрицы с положительными элементами на диагонали. Обозначим группу $\prod_{K_{i}} A\left(k, K_{i}\right)$ через $A$, группу $\prod_{K_{i}} N\left(k, K_{i}\right)$ через $N$. Разложение Ивасава дает разложение $G=A N O$. Пусть $d a^{(i)}$ - мера Хаара на $A\left(k, K_{i}\right)$ равная $1 /\left(a_{1} \cdots a_{k}\right) d a_{1} \cdots d a_{k}$, где $a_{i}$ есть $i$-й диагональный элемент. После замены переменной $a_{i}=\mathrm{e}^{e_{i}}$ получим $d e_{1} \cdots d e_{k}$. Пусть $d n^{(i)}$ - мера Хаара на $N\left(k, K_{i}\right)$, равная стандартной мере Лебега на $\mathbb{R}^{k(k-1) \operatorname{dim}\left(K_{i}\right) / 2}$. Mера $d g^{(i)}$ на $\operatorname{Gl}\left(n, K_{i}\right)$, равная $\rho_{K_{i}}(a) d a^{(i)} d n^{(i)} d o^{(i)}$, где $\rho_{K_{i}}(a)=\prod_{k<l}\left(\frac{a_{k}}{a_{l}}\right)^{\operatorname{dim}_{\mathbb{R}}\left(K_{i}\right)}$ и $d o^{(i)}-$ произвольная мера Хаapa на $O\left(K_{i}\right)$, является мерой Хаара [2; лемма 3]. Положим $\rho=\prod_{K_{l}} \rho_{K_{l}}$, через $d n$ и $d a$ обозначим меры Хаара на $N$ и $A$ соответственно. В разложении Ивасава для группы $G^{\prime}$ группа $A$ заменяется на подгруппу $A^{\prime} \subset A$, состоящую из наборов диагональных матриц $\left(P^{i}\right)$ таких, что $\prod_{K_{i}} \operatorname{det}\left(P^{i}\right)^{\operatorname{dim}_{\mathbb{R}}\left(K_{i}\right)}=1$. Пусть $d a^{\prime}-$ произвольная
мера Хаара на $A^{\prime}$.

ОПРедЕЛЕНиЕ 3 . Пусть $X$ - пространство с левым действием группы $G$. Мера $\mu$ на $X$ называется $G$-эквивариантной, если $g_{*} \mu=\mu$ для любого элемента $g \in G$.

Tеорема 1. Мера $\rho(a) d a^{\prime} d n$ является $G^{\prime}$-эквивариантной на $G^{\prime} / O$.

Далее всюду $k=2$. Пусть $a, b-$ базис модуля $F^{2}$.

Работа выполнена при поддержке гранта MK-4881.2011.1, Лаборатории алгебраической геометрии ГУ-ВШЭ, гранта правительства РФ дог. 1111.G34.31.0023, гранта РФФИ 11-01-00145а и программы "Ведущие научные школы" (грант НШ-4713.2010.1). 
ОПредЕлЕниЕ 4. Назовем главными нестабильными решетками те специальные решетки, члены канонической фильтрации которых изоморфны $F$.

Следующая лемма описывает фундаментальную область $G^{\prime} / O$ относительно действия $H$, из которой легко выделяются главные нестабильные решетки.

Лемма 1. Элементы $G / O$ представимы набором верхнетреугольных матрии, $\left(\left(\begin{array}{cc}a_{i} & 0 \\ 0 & b_{i}\end{array}\right)\left(\begin{array}{cc}1 & c_{i} \\ 0 & 1\end{array}\right)\right)$. В качестве фундаментальной области для действия $H$ на $G / O$ можно взять наборы верхнетреугольных матрии, для которых выполняются нижеперечисленные условия:

1) элемент $a \in F^{2}$ выбран так, чтобъ кообъем $\operatorname{covol}(F a) \subset \mathbb{R}^{n}=\prod K_{i}$ был наименьшим среди всех элементов $F^{2}$, дополнимых до базиса;

2) ортогональные проекции наборов $\left\{\ln \left(a_{i} / b_{i}\right)\right\}$ u $\left\{\ln \left(a_{i} b_{i}\right)\right\}$ на плоскость, ортогональную вектору $\left(\operatorname{dim}\left(K_{1}\right), \ldots, \operatorname{dim}\left(K_{r+s}\right)\right)$, лежат в некоторой фиксированной фундаментальной области $A_{0}$ для $F^{*}$;

3) набор $\left\{c_{i}\right\}$ лежит в некоторой фиксированной фундаментальной области $N_{0}$ для аддитивного действия $F$ на пространстве $\prod K_{i}$.

Обозначим $e_{i}^{1}=\ln a_{i}, e_{i}^{2}=\ln b_{i}$. Пусть также $r_{i}=e_{i}^{1}-e_{i}^{2}, r=\sum \operatorname{dim}_{\mathbb{R}}\left(K_{i}\right) r_{i}$ и $f_{i}=e_{i}^{1}+e_{i}^{2}, f=\sum \operatorname{dim}_{\mathbb{R}}\left(K_{i}\right) f_{i}$. Заметим, что $\rho(a)=\mathrm{e}^{r}$. Пусть $r^{\prime}$ и $f^{\prime}-$ opтонормированные координаты в плоскостях $\sum \operatorname{dim}_{\mathbb{R}}\left(K_{i}\right) r_{i}=0$ и $\sum \operatorname{dim}_{\mathbb{R}}\left(K_{i}\right) f_{i}=0$ соответственно. Возьмем в $A^{\prime}$ меру Хаара $d a^{\prime \prime}$, равную $d r d r^{\prime} d f^{\prime}$. Меру на вложенных решетках $\mathrm{e}^{r} d a^{\prime \prime} d n$ обозначим $\nu_{G^{\prime} / O}^{2}$.

ТеОрема 2. Для меры $\nu_{G^{\prime} / O}^{2}$ объем главных нестабильных решеток ранга 2 равен $R_{K}^{2} D_{K}^{1 / 2}$, где $R_{K}$ и $D_{K}$ - регулятор и дискриминант числового поля.

Объем фундаментального множества для группы $H$ внутри множества вложенных решеток ранга 2 с кообъемом, не превосходящим 1 , относительно $G^{\prime}$-эквивариантной меры $4^{r+s} \rho(a) \prod_{K_{i}} \mathrm{e}^{3 f_{i} \operatorname{dim}_{\mathbb{R}}\left(K_{i}\right)} d a d n$ равен $2^{r-2 s} R_{K} D_{K}^{3 / 2} \zeta_{K}(2) / 3$. Это следствие формулы Зигеля (см. [3], а также обзор [4]) для $k=2$. С помощью вычислений с мерами Хаара отсюда можно вывести следующую теорему.

Теорема 3. Объем фундаментального множества $M$ по мере $\nu_{G^{\prime}}^{2} /$ о равен $2^{-3 s} \times$ $(r+4 s) R_{K} D_{K}^{3 / 2} \zeta(2)$.

СлЕДСтвиЕ 1. Имеет место неравенство $2^{-3 s}(r+4 s) D_{K} \zeta_{K}(2) \geqslant R_{K}$.

ДокАзАтельство. Сравниваем объем главных нестабильных решеток из теоремы 2 с объемом фундаментального множества $M$.

Автор благодарен А.Н. Паршину за постановку задачи и многочисленные обсуждения, а также М. А. Цфасману за помощь с оценками регулятора и дискриминанта.

\section{Список литературы}

[1] D. R. Grayson, Comment. Math. Helv., 59 (1984), 600-634. [2] А. Борель, Математика, 12:4 (1968), 80-103. [3] C. L. Siegel, Trans. Amer. Math. Soc., 39 (1936), 209-218. [4] A. H. Паршин, Итоги науки и техники. Соврем. матем. и ее прил., 70, ВИНИТИ, М., 2001, 159-164.

\section{P. Я. Будылин (R. Ya. Budylin)}

Московский государственный университет им. М. В. Ломоносова

E-mail: budylin@yandex.ru
Представлено Э. Б. Винбергом Принято редколлегией 18.05.2011 